\title{
Experimental multiparticle entanglement dynamics induced by decoherence
}

\author{
Julio T. Barreiro ${ }^{1 \star}$, Philipp Schindler ${ }^{1}$, Otfried Gühne ${ }^{2,3,4 \star}$, Thomas Monz ${ }^{1}$, Michael Chwalla', \\ Christian F. Roos ${ }^{1,2}$, Markus Hennrich ${ }^{1}$ and Rainer Blatt ${ }^{1,2}$
}

\begin{abstract}
Multiparticle entanglement leads to richer correlations than two-particle entanglement and gives rise to striking contradictions with local realism ${ }^{1}$, inequivalent classes of entanglement ${ }^{2}$ and applications such as one-way or topological quantum computing $^{3,4}$. When exposed to decohering or dissipative environments, multiparticle entanglement yields subtle dynamical features and access to new classes of states and applications. Here, using a string of trapped ions, we experimentally characterize the dynamics of entanglement of a multiparticle state under the influence of decoherence. By embedding an entangled state of four qubits in a decohering environment (through spontaneous decay), we observe a rich dynamics crossing distinctive domains: Bell-inequality violation, entanglement superactivation, bound entanglement and full separability. We also develop new theoretical tools for characterizing entanglement in quantum states. Recent quantum-computing, state-engineering and simulation paradigms driven by dissipative or decohering environments ${ }^{5-7}$ can benefit from the environment engineering techniques demonstrated here.
\end{abstract}

When exposed to an environment, bipartite entanglement already shows subtle dynamical features, for example, finite-time disentanglement ${ }^{8,9}$. In a multipartite setting, decoherence and dissipation enable new quantum applications ${ }^{5-7}$, induce diverse dynamics because of the robustness of the different classes of states ${ }^{10}$ and can decrease the number of particles genuinely entangled-an effect recently observed ${ }^{11}$. However, decoherence can also influence other state properties useful for quantum information processing, such as distillability. A state is distillable if, using local operations and classical communication, one can extract the maximally entangled states required by several quantum communication protocols such as teleportation ${ }^{12}$.

An environment can also drive a multiparticle entangled and distillable state into the undistillable but still entangled domain ${ }^{13,14}$, called bound entangled ${ }^{15}$. This class of states is expected to appear in many-body systems ${ }^{16}$, and despite being undistillable, it is useful for entanglement superactivation ${ }^{17}$, quantum secret sharing ${ }^{18}$ or remote information concentration ${ }^{19}$. Bound entangled states have also given insights into classical information theory: a classical analogue of bound entanglement, called bound information, exists ${ }^{20}$. Recently, a bound entangled state was simulated with photons ${ }^{21}$, as well as a variant called pseudo-bound entanglement, using NMR (ref. 22).

Here, we report the experimentally observed dynamics of entanglement and distillability in the neighbourhood of a bound entangled state under a partially decohering environment. Entanglement and distillability of a multiparty system are defined with respect to the state bipartitions, or abstract splits into two subsystems. Our work focuses on a four-party system that can be bi-partitioned in two ways, either in pairs $(2: 2)$, or as a single party plus the rest (1:3). In this case, we call the state of the four particles 2:2 (1:3) separable if every 2:2 (1:3) bipartition can be written as a mixture of bipartite states $\left|\psi_{\mathrm{k}}\right\rangle$ as

$$
\rho=\sum_{\mathrm{k}} p_{\mathrm{k}}\left|\psi_{\mathrm{k}}\right\rangle\left\langle\psi_{\mathrm{k}}|, \quad| \psi_{k}\right\rangle=\left|\eta_{\alpha \beta}^{(\mathrm{k})}\right\rangle\left|\eta_{\mu \nu}^{(\mathrm{k})}\right\rangle\left(\left|\eta_{\alpha}^{(\mathrm{k})}\right\rangle\left|\eta_{\beta \mu \nu}^{(\mathrm{k})}\right\rangle\right)
$$

and $\alpha, \beta, \mu$ and $v$ denote the particles. Otherwise, if every 2:2 (1:3) bipartition cannot be written as above, we call the state $2: 2(1: 3)$ entangled. Regarding distillability, a state is 2:2 (1:3) distillable if, for every 2:2 (1:3) bipartition, a Bell pair can be distilled, each element of which belongs to one subsystem. An even stronger distillability property is entanglement superactivation, which in the case of a four-particle state, enables five parties (A, B, C, D and $\mathrm{E})$ sharing two copies of the state $\left(\rho_{\mathrm{ABCD}}\right.$ and $\left.\rho_{\mathrm{ABCE}}\right)$ to distil entanglement between the two parties holding a single particle (D and E; ref. 17).

Our study starts by preparing a 2:2- and 1:3-entangled state, violating a Clauser-Horne-Shimony-Holt (CHSH)-type Bell inequality ${ }^{18}$, and capable of entanglement superactivation. As we apply a tunable decohering environment, the state stops violating the Bell inequality. Then, only within a region of further decoherence is the entanglement superactivation protocol successful, and the 2:2 and 1:3 entanglement is preserved with even more decoherence. Increasing the decoherence eventually eliminates the entanglement in both bipartitions at different, but finite, times. This finite-time disentanglement behaviour is

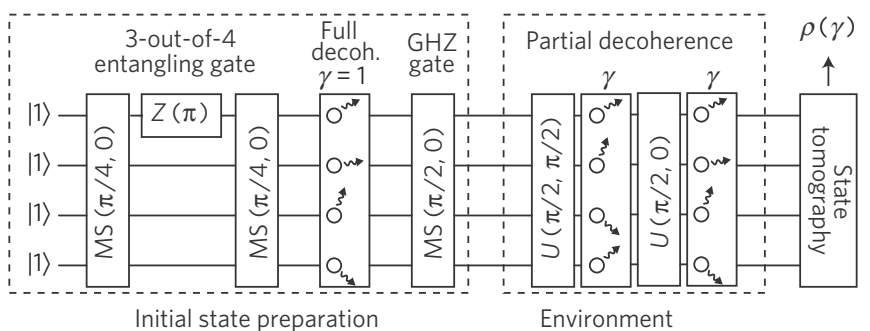

Figure 1 | Experimental sequence for studying the dynamics of multiparticle entanglement and distillability induced by decoherence. The operations indicated in the sequence are collective unitary transformations $U(\theta, \phi)$, light-shift operations $Z(\theta)$ and entangling operations $\operatorname{MS}(\theta, \phi)$.

\footnotetext{
${ }^{1}$ Institut für Experimentalphysik, Universität Innsbruck, Technikerstr. 25, 6020 Innsbruck, Austria, ${ }^{2}$ Institut für Quantenoptik und Quanteninformation, Österreichische Akademie der Wissenschaften, Technikerstr. 21A, 6020 Innsbruck, Austria, ${ }^{3}$ Institut für Theoretische Physik, Universität Innsbruck, Technikerstr. 25, 6020 Innsbruck, Austria, ${ }^{4}$ Fachbereich Physik, Universität Siegen, Walter-Flex-Str. 3, D-57068 Siegen, Germany. *e-mail: Julio.Barreiro@uibk.ac.at; Otfried.Guehne@uibk.ac.at.
} 


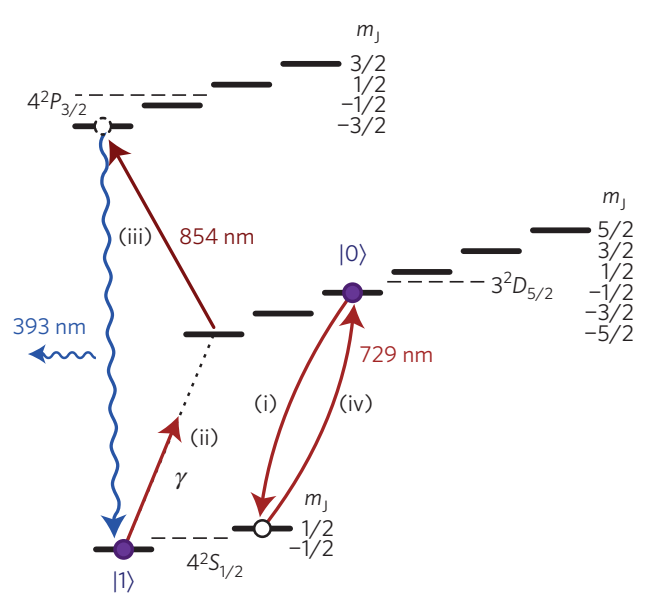

Figure 2 | Zeeman-split ${ }^{40} \mathrm{Ca}^{+}$levels for the implementation of tunable decoherence through entanglement with a spontaneously decaying photon. Partial decoherence is realized by simultaneously carrying out on all ions the steps: (i) hiding of $|0\rangle$, (ii) partial transfer of $|1\rangle$, (iii) quenching and decay of $\sqrt{\gamma}|1\rangle$, and finally (iv) restoring $|0\rangle$.

also sometimes called environment-induced sudden death of entanglement ${ }^{8,9}$. As the $2: 2$ entanglement disappears before the 1:3 entanglement, we realize a domain that can be called bound entangled $^{13}$. Similarly, a recent theoretical study showed that a four-qubit Greenberger-Horne-Zeilinger ${ }^{1}$ (GHZ) state can decay into a bound entangled state by becoming 2:2 undistillable although slightly 1:3 entangled ${ }^{13}$. Further decoherence eventually makes the state fully separable, long before the single-particle coherence asymptotically disappears.

Our experiment proceeds in three stages: state preparation, exposure to tunable decoherence and state characterization. The goal of the first stage is to generate a state that decays into the domain of bound entangled states when exposed to a partially decohering mechanism. We chose to prepare an initial state close to the Smolin state $\rho_{\mathrm{S}}$ (ref. 23), a known four-qubit bound entangled state usually written as a mixture of the Bell states $\Phi^{ \pm}=(|00\rangle \pm|11\rangle) / \sqrt{2}$ and $\Psi^{ \pm}=(|01\rangle \pm|10\rangle) / \sqrt{2}$ as

$$
\rho_{\mathrm{S}}=\left[\left|\Phi^{+} \Phi^{+}\right\rangle\left\langle\cdot|+| \Phi^{-} \Phi^{-}\right\rangle\left\langle\cdot|+| \Psi^{+} \Psi^{+}\right\rangle\left\langle\cdot|+| \Psi^{-} \Psi^{-}\right\rangle\langle\cdot|\right] / 4
$$

where $|0\rangle$ and $|1\rangle$ are the qubit basis states, and we use the notation $|\chi\rangle\langle\cdot|\equiv| \chi\rangle\langle\chi|$. The initial-state preparation sequence, shown in
Fig. 1, follows from realizing that the Smolin state is a mixture of four GHZ-like states

$$
\begin{aligned}
\rho_{\mathrm{S}}= & {[(|1111\rangle+|0000\rangle)\langle\cdot|+(|1100\rangle+|0011\rangle)\langle\cdot|} \\
& +(|1010\rangle+|0101\rangle)\langle\cdot|+(|1001\rangle+|0110\rangle)\langle\cdot|] / 8
\end{aligned}
$$

Following the steps in Fig. 1 in reverse order, this state can be reached by applying a single-step GHZ-entangling operation to the mixture

$$
\rho=[|1111\rangle\langle\cdot|+| 1100\rangle\langle\cdot|+| 1010\rangle\langle\cdot|+| 1001\rangle\langle\cdot|] / 4
$$

where the operation takes a state of the form $\left|x_{1} x_{2} x_{3} x_{4}\right\rangle$ into $\left(\left|x_{1} x_{2} x_{3} x_{4}\right\rangle+\left|\bar{x}_{1} \bar{x}_{2} \bar{x}_{3} \bar{x}_{4}\right\rangle\right) / \sqrt{2}$ and $\bar{x}_{i}$ denotes the complement of state $x_{i}$ of ion $i$ in the computational basis. The mixture described by equation (1), in turn, can be generated by completely decohering a state in which three out of four particles are entangled,

$$
|\Psi\rangle=(|1111\rangle+|1100\rangle+|1010\rangle+|1001\rangle) / 2
$$

with the same mechanism as used in the second stage of our study. Finally, another GHZ-entangling operation and an NMR-like refocusing technique ${ }^{24}$ applied to the state $|1111\rangle$ generates the state in equation (2).

In the second stage, the intended rich dynamics was achieved by increasingly decohering the initial state, as described below (see also Supplementary Information). We characterized the state's entanglement and distillability in the last stage. A single criterion, the Peres-Horodecki separability criterion ${ }^{25}$, can prove undistillability, and its extension into a measure, known as negativity ${ }^{26}$, can quantify entanglement. According to this criterion, if a state is separable then its partial transposition has no negative eigenvalues (it has a positive partial transpose, PPT). On the other hand, it has been shown that PPT states are undistillable ${ }^{15}$. Therefore, entangled but undistillable states, or bound entangled states, can be detected by verifying that every 1:3 bipartition has a negative partial transpose (entanglement) ${ }^{27}$, whereas every 2:2 bipartition has a PPT (undistillability) ${ }^{15}$. To determine the state's undistillability properties, we carried out a complete tomographic reconstruction. Full knowledge of the state enabled us to also check further separability and distillability properties. Especially, we designed a new algorithm to prove separability of the states, which is a stronger statement than undistillability (see Supplementary Information).

Our work was carried out on a system of four ${ }^{40} \mathrm{Ca}^{+}$ions confined to a string by a linear Paul trap with axial (radial) $|\rho(\gamma)|$ for $\gamma=0$

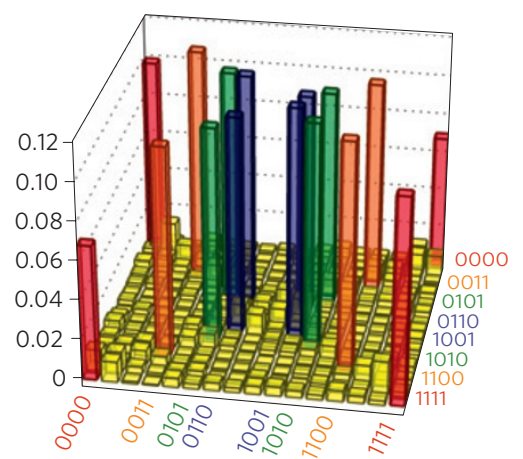

$|\rho(\gamma)|$ for $\gamma=0.32$

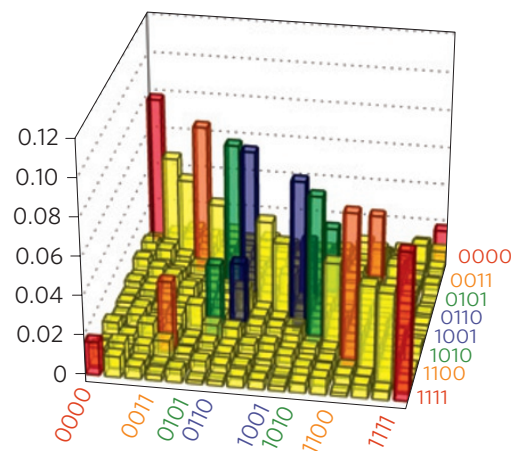

$|\rho(\gamma)|$ for $\gamma=0.60$

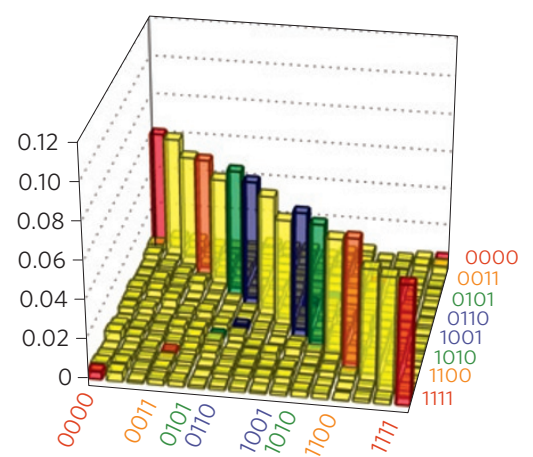

Figure 3 | Density matrices (absolute value) of states that are 2:2 and 1:3 entangled $(\gamma=0)$, bound entangled $(\gamma=0.32)$ and fully separable $(\gamma=\mathbf{0 . 6 0})$. The four GHZ-like components of the Smolin state $[(|0000\rangle+|1111\rangle)\langle\cdot|+(|0011\rangle+|1100\rangle)\langle\cdot|+(|0101\rangle+|1010\rangle)\langle\cdot|+(|0110\rangle+|1001\rangle)\langle\cdot|] / 8$ are highlighted with distinctive colours. The density matrices of all measured states are shown in the Supplementary Information. 

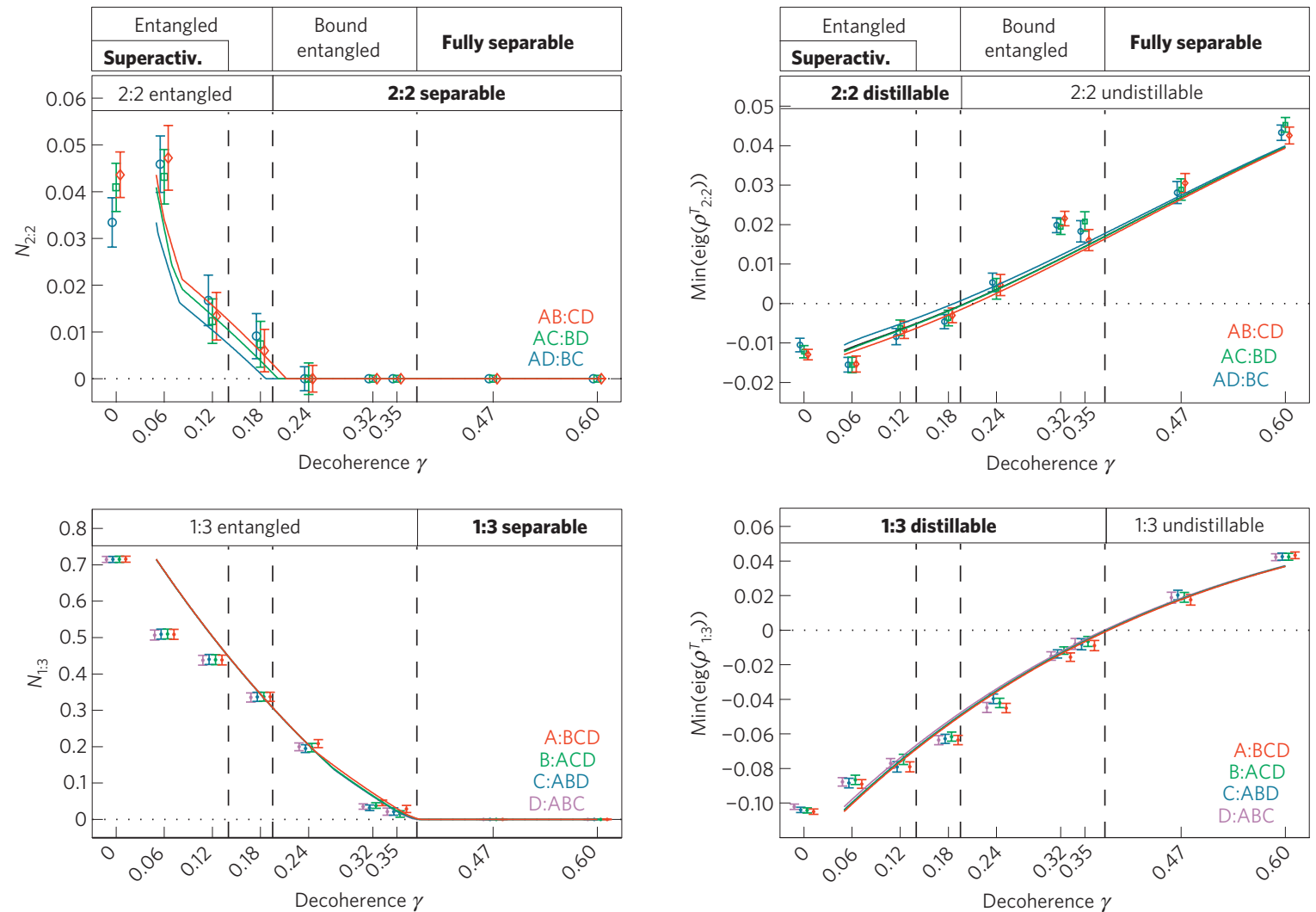

Figure 4 | Negativity and smallest eigenvalue of the partial transpose for each 2:2 and 1:3 bipartition of the measured states as a function of decoherence. A positive smallest eigenvalue of the partial transpose, $\min \left[\operatorname{eig}\left(\rho^{T}\right)\right]>0$, reveals undistillability. Bipartition data are slightly offset horizontally for clarity, but all visible groups correspond to the same amount of decoherence indicated by the tick marks. The error bars indicate \pm 1 standard deviation, calculated from propagated statistics in the raw state identification events. The solid lines were calculated by decohering the prepared initial state with a 0.05 offset in $\gamma$ (owing to imperfections in the decoherence implementation). The properties shown in bold were determined by tests independent of the plotted data (see Supplementary Information).

Table 1 | Negativity and smallest eigenvalue of the partial transpose of representative states.

\begin{tabular}{|c|c|c|c|c|c|c|c|c|c|}
\hline & & $A B: C D$ & $A C: B D$ & $A D: B C$ & & $A: B C D$ & B:ACD & C:ACD & D:ABC \\
\hline$\gamma=0$ & $N_{2: 2}=$ & $0.033(5)$ & $0.041(5)$ & 0.04 & $N_{1: 3}=$ & 0.71 & $0.715(8)$ & $0.715(8)$ & $0.716(8)$ \\
\hline$\gamma=0.32$ & $\min \left[\operatorname{eig}\left(\rho^{T_{2: 2}}\right)\right]=$ & $0.020(2)$ & $0.019(2)$ & $0.022(2)$ & $N_{1: 3}=$ & $0.035(7)$ & $0.032(8)$ & $0.038(8)$ & $0.045(7)$ \\
\hline$\gamma=0.47$ & $\min \left[\operatorname{eig}\left(\rho^{T_{2: 2}}\right)\right]=$ & $0.028(3)$ & $0.029(3)$ & $0.031(2)$ & $\min \left[\operatorname{eig}\left(\rho^{T_{1: 3}}\right)\right]=$ & $0.019(3)$ & $0.020(3)$ & $0.019(3)$ & $0.018(3)$ \\
\hline$\gamma^{\prime}=0.43$ & $\min \left[\operatorname{eig}\left(\rho^{T_{2: 2}}\right)\right]=$ & $0.013(2)$ & $0.011(2)$ & $0.013(2)$ & $N_{1: 3}=$ & $0.038(7)$ & $0.039(7)$ & $0.042(7)$ & $0.045(7)$ \\
\hline
\end{tabular}

The parties in the bipartitions are labelled as A, B, C and D. The state with $\gamma^{\prime}=0.43$ was prepared by decohering in a single step, see text. Uncertainties in parentheses indicate one standard deviation, calculated from propagated statistics in the raw state identification events.

vibrational frequencies of approximately $1.2 \mathrm{MHz}(4.4 \mathrm{MHz})$. Each ion hosts a qubit on the electronic Zeeman levels $D_{5 / 2}(m=-1 / 2)$, encoding $|0\rangle$, and $S_{1 / 2}(m=-1 / 2)$, encoding $|1\rangle$, determined by a magnetic field of $\approx 4 \mathrm{G}$. The ion string was optically pumped to the starting quantum state $|1111\rangle$ after being Doppler cooled and sideband cooled to the ground state of the axial centre-of-mass mode ${ }^{28}$ (Supplementary Information). The state of the qubits can be manipulated through (i) collective unitary operations $U(\theta, \phi)=\exp \left(-i(\theta / 2) S_{\phi}\right)$, with $S_{\phi}=\sum_{k=1}^{4} \sigma_{\phi}^{(\mathrm{k})}, \sigma_{\phi}^{(\mathrm{k})}=\cos (\phi) \sigma_{x}^{(\mathrm{k})}+\sin (\phi) \sigma_{y}^{(\mathrm{k})}$ and $\sigma_{n}^{(\mathrm{k})}$ being a Pauli spin operator acting on the $k$ th ion, (ii) single-qubit light-shift operations $Z^{(\mathrm{k})}(\theta)=\exp \left(-i(\theta / 2) \sigma_{z}^{(\mathrm{k})}\right)$ and (iii) a GHZ-entangling operation known as a Mølmer-Sørensen gate ${ }^{29,30}$, $\operatorname{MS}(\theta, \phi)=\exp \left(-i(\theta / 4) S_{\phi}^{2}\right)$. We can prepare four-qubit GHZ states with a fidelity of $96 \%$ and carry out collective unitaries and light-shift operations at a fidelity of $99 \%$. These imperfections determine the proximity of our prepared initial state to the Smolin state. The full experimental sequence is shown in Fig. 1.

The partially decohering mechanism indicated in Fig. 1 was implemented in the four steps shown in Fig. 2: (i) hiding the population in $|0\rangle$ by a full coherent transfer into $S_{1 / 2}(m=1 / 2)$; (ii) transfer of the population in $|1\rangle$ into the superposition $\sqrt{1-\gamma}|1\rangle+$ $\sqrt{\gamma}\left|D_{5 / 2}(m=-5 / 2)\right\rangle$; (iii) quenching of the population in $D_{5 / 2}(m=-5 / 2)$ into $P_{3 / 2}(m=-3 / 2)$ by exposure to $854 \mathrm{~nm}$ radiation, so that it spontaneously decays to $|1\rangle$; and finally (iv) restoring the hidden population into $|0\rangle$ (for other techniques see ref. 31). In this way, a fraction $\gamma$ of the population in $|1\rangle$ irreversibly loses phase coherence with $|0\rangle$ by tracing over the emitted photon. In this case we call this basis-dependent partial loss of coherence decoherence in the $|0\rangle,|1\rangle$ basis. In our experiment, we decohere the states in the $|0\rangle \pm|1\rangle$ and $|0\rangle \pm i|1\rangle$ bases by applying the collective unitary rotations $U(\pi / 2, \pi / 2)$ and $U(\pi / 2,0)$ before the above 
decoherence, as shown in Fig. 1. This decoherence also drives a GHZ state into a narrow bound entangled domain (see Supplementary Information). The complete decohering step in the preparation of the intermediate state in equation (1) was carried out in the computational basis with $\gamma=1$.

The dynamics of entanglement was explored by varying the amount of decoherence $\gamma$ to which the initial state was exposed (see Fig. 1). After being partially decohered, the density matrices of the prepared states $\rho(\gamma)$ were tomographically reconstructed (see Fig. 3). Error analysis was carried out using Monte Carlo simulations over the raw data outcomes of the state tomography. The amount of entanglement and signature of undistillability of the measured states as a function of decoherence $\gamma$ are shown in Fig. 4; the explicit values for the most representative states are quoted in Table 1. Figure 4 also indicates other properties of the states determined independently of the plotted data (full details in Supplementary Information).

The measured initial state $(\gamma=0)$ is highly entangled in the $1: 3$ bipartitions $\left(N_{1: 3} \gg 0\right)$ and slightly entangled in the 2:2 $\left(N_{2: 2}>0\right)$; in addition, it violates a CHSH-type Bell inequality and is capable of entanglement superactivation. The properties of the state already change at $\gamma=0.06$, when the state no longer violates the tested Bell inequality. The entanglement superactivation protocol is successful in the domain of states from $\gamma=0$ up to $\gamma=0.12$. We show strong evidence in the Supplementary Information that all measured states from $\gamma=0$ to $\gamma=0.18$ are biseparable. This means that, although they are entangled with respect to any fixed 1:3 and 2:2 bipartition, they can be written as a mixture of separable states, which are separable with respect to different bipartitions.

The passage into bound entanglement occurs at $\gamma \approx 0.21$. Although the measured state at $\gamma=0.24$ is $2: 2$ separable and 1:3 entangled, the bound entanglement is arguable because a fraction of the Monte Carlo samples revealed 2:2 entanglement, thus indicating insufficient statistics. By $\gamma=0.32$, the state is now bona fide bound entangled, when also all Monte Carlo samples are 1:3 entangled, 2:2 undistillable, and even 2:2 separable. The change into full undistillability is heralded by the state measured at $\gamma=0.47$ because all eigenvalues of the partial transpose were positive for every bipartition, as shown in Table 1 and Fig. 4 . However, although the measured state is fully separable, known methods failed to prove the separability of the Monte Carlo samples. By $\gamma=0.60$, we achieve full separability in the measured data and all Monte Carlo samples.

We also found a bound entangled state by decohering the initial state in only the $|0\rangle \pm|1\rangle$ basis, represented by the state at $\gamma^{\prime}=0.43$ (see Table 1). We thus controllably realized a passage into bound entanglement in a simple decohering environment, with statistically significant entanglement in the 2:2 partitions and positivity of the eigenvalues of the 1:3 partially transposed states.

We experimentally explored the dynamics of multiparticle entanglement, separability and distillability under a tunable decohering mechanism. The influence of the environment naturally created a bound entangled state. Our investigation on the dynamics of multiparticle entanglement can be extended to observe bound entanglement on other states, such as decaying GHZ states ${ }^{13}$ or thermal states of spin models ${ }^{16}$. In addition, our universal set of gates, decoherence mechanism and dissipation via optical pumping form a toolset for demonstrating new quantum-computing, stateengineering and simulation paradigms ${ }^{5-7}$.

Received 7 May 2010; accepted 10 August 2010; published online 26 September 2010

\section{References}

1. Greenberger, D. M., Horne, M. A. \& Shimony, A. Bell's theorem without inequalities. Am. J. Phys. 58, 1131-1143 (1990).

2. Dür, W., Vidal, G. \& Cirac, J. Three qubits can be entangled in two inequivalent ways. Phys. Rev. A 62, 062314 (2000).
3. Raussendorf, R. \& Briegel, H. J. A one-way quantum computer. Phys. Rev. Lett. 86, 5188-5191 (2001).

4. Nayak, C. et al. Non-Abelian anyons and topological quantum computation. Rev. Mod. Phys. 80, 1083-1159 (2008).

5. Diehl, S. et al. Quantum states and phases in driven open quantum systems with cold atoms. Nature Phys. 4, 878-883 (2008).

6. Verstraete, F., Wolf, M. M. \& Cirac, J. I. Quantum computation and quantum-state engineering driven by dissipation. Nature Phys. 5, 633-636 (2009).

7. Weimer, H. et al. A Rydberg quantum simulator. Nature Phys. 6, 382-388 (2010).

8. Yu, T. \& Eberly, J. Sudden death of entanglement. Science 323, 598-601 (2009).

9. Almeida, M. et al. Environment-induced sudden death of entanglement. Science 316, 579-582 (2007)

10. Dür, W. \& Briegel, H-J. Stability of macroscopic entanglement under decoherence. Phys. Rev. Lett. 92, 180403 (2004).

11. Papp, S. et al. Characterization of multipartite entanglement for one photon shared among four optical modes. Science 324, 764-768 (2009).

12. Bennett, C. H. et al. Teleporting an unknown quantum state via dual classical and Einstein-Podolsky-Rosen channels. Phys. Rev. Lett. 70, 1895-1899 (1993).

13. Aolita, L. et al. Scaling laws for the decay of multiqubit entanglement. Phys. Rev. Lett. 100, 080501 (2008).

14. Borras, A. et al. Robustness of highly entangled multiqubit states under decoherence. Phys. Rev. A 79, 022108 (2009).

15. Horodecki, M., Horodecki, P. \& Horodecki, R. Mixed-state entanglement and distillation: Is there a 'bound' entanglement in nature? Phys. Rev. Lett. 80, 5239-5242 (1998).

16. Tóth, G. et al. Optimal spin squeezing inequalities detect bound entanglement in spin models. Phys. Rev. Lett. 99, 250405 (2007).

17. Shor, P. W., Smolin, J. A. \& Thapliyal, A. V. Superactivation of bound entanglement. Phys. Rev. Lett. 90, 107901 (2003).

18. Augusiak, R. \& Horodecki, P. Generalized Smolin states and their properties. Phys. Rev. A 73, 012318 (2006).

19. Murao, M. \& Vedral, V. Remote information concentration using a bound entangled state. Phys. Rev. Lett. 86, 352-355 (2001).

20. Acín, A., Cirac, J. I. \& Masanes, L. Multipartite bound information exists and can be activated. Phys. Rev. Lett. 92, 107903 (2004).

21. Amselem, E. \& Bourennane, M. Experimental four-qubit bound entanglement. Nature Phys. 5, 748-752 (2009).

22. Kampermann, H. et al. Experimental generation of pseudo-bound-entanglement. Phys. Rev. A 81, 040304(R) (2010).

23. Smolin, J. A. Four-party unlockable bound entangled state. Phys. Rev. A 63, 032306 (2001).

24. Vandersypen, L. \& Chuang, I. NMR techniques for quantum control and computation. Rev. Mod. Phys. 76, 1037-1069 (2005).

25. Peres, A. Separability criterion for density matrices. Phys. Rev. Lett. 77, 1413-1415 (1996).

26. Vidal, G. \& Werner, R. F. Computable measure of entanglement. Phys. Rev. A 65, 032314 (2002).

27. Dür, W., Cirac, J. I. \& Tarrach, R. Separability and distillability of multiparticle quantum systems. Phys. Rev. Lett. 83, 3562-3565 (1999).

28. Schmidt-Kaler, F. et al. How to realize a universal quantum gate with trapped ions. Appl. Phys. B 77, 789-796 (2003).

29. Mølmer, K. \& Sørensen, A. Multi-particle entanglement of hot trapped ions. Phys. Rev. Lett. 82, 1835-1838 (1999).

30. Sackett, C. A. et al. Experimental entanglement of four particles. Nature 404, 256-259 (2000).

31. Myatt, C. J. et al. Decoherence of quantum superpositions through coupling to engineered reservoirs. Nature 403, 269-273 (2000).

\section{Acknowledgements}

We gratefully acknowledge support by the Austrian Science Fund, the European Commission (SCALA, NAMEQUAM, QICS), the Institut für Quanteninformation $\mathrm{GmbH}$ and a Marie Curie International Incoming Fellowship within the 7th European Community Framework Programme. This material is based on work supported in part by Intelligence Advanced Research Projects Activity.

\section{Author contributions}

J.T.B., P.S., T.M. and M.C. carried out the experiment; O.G. provided the theoretical part and partially analysed the data; J.T.B. conceived the experiment and analysed the data; R.B., J.T.B., P.S., T.M., M.C., C.F.R. and M.H. contributed to the experimental set-up; and all authors co-wrote the paper.

\section{Additional information}

The authors declare no competing financial interests. Supplementary information accompanies this paper on www.nature.com/naturephysics. Reprints and permissions information is available online at http://npg.nature.com/reprintsandpermissions. Correspondence and requests for materials should be addressed to J.T.B. or O.G. 